\title{
0 objeto de trabalho na Estratégia Saúde da Família
}

\author{
Geraldo Eduardo Guedes de Brito(a) \\ Antonio da Cruz Gouveia Mendes ${ }^{(b)}$ \\ Pedro Miguel dos Santos Neto ${ }^{(c)}$
}

Brito GEG, Mendes ACG, Santos Neto PM. Purpose of work in the Family Health Strategy. Interface (Botucatu). 2018; 22(64):77-86.

This study aimed to analyze the Family Health Strategy workers' perception related with the pupose of their work. Twelve interviews were conducted: nine with workers from three Family Health Teams, that were considered as reference by municipal managers, and three with workers from Family Health Support Group, all of them with higher education. The transcription of the interviews was submitted to content analysis. Conceptual construction of the interviewed workers' purpose of work was identified as regardingthe recognition of individualcentered care related with their unique needs, associated with their families and communities. This result indicated the high degree of complexity that guides the work under the Family Health Strategy, where the object of work goes beyond the individual and the biological dimension, and encompasses the social determination and its familiar and communal relationship.

Keywords: Family Health Strategy. Work. Human resources. Qualitative analysis.
Este estudo objetivou analisar a percepção de trabalhadores da Estratégia Saúde da Família acerca de seu objeto de trabalho. Foram realizadas 12 entrevistas: nove com trabalhadores de três Equipes Saúde da Família, consideradas de referência por gestores municipais, e três com trabalhadores do Núcleo de Apoio à Saúde da Família destas, todos com nível superior. A transcrição das entrevistas foi submetida à análise de conteúdo. Foi identificada a construção conceitual do objeto de trabalho dos entrevistados. Tratou-se do reconhecimento da produção do cuidado centrada no indivíduo a partir de suas necessidades singulares, associado a sua família e comunidade. Esse resultado apontou o elevado grau de complexidade que orienta o trabalho no âmbito da Saúde da Família, onde o objeto de trabalho extrapola a dimensão individual e biologicista, assumindo a determinação social e a sua relação familiar e comunitária.

Palavras-chave: Estratégia Saúde da Família. Trabalho. Recursos humanos. Análise qualitativa.

\footnotetext{
(a) Departamento de Fisioterapia, Centro de Ciências da Saúde, Universidade Federal da Paraíba. Campus I, Jardim Universitário, s/n, Castelo Branco. João Pessoa, PB, Brasil. 58059-900. eduardo.guedes.ufpb@ gmail.com

(b, c) Departamento de Saúde Coletiva, Instituto

Aggeu Magalhães (Fiocruz-PE). Recife, PE, Brasil. amendes@ cpqam.fiocruz.

br; pedromiguel@ cpqam.fiocruz.br
} 


\section{Introdução}

A Estratégia Saúde da Família (ESF) é o modelo preferencial de organização da Atenção Primária à Saúde (APS) no Brasil, e espera-se que ela seja capaz de abordar o processo de saúde-doença dos indivíduos de modo singular e articulado ao contexto familiar e comunitário. Nas últimas duas décadas, a ESF ampliou significativamente o acesso aos serviços de atenção à saúde. Para dimensionalizarmos essa questão, cabe citar que, em janeiro de 2000, havia 4.563 Equipes Saúde da Família (EqSF) implantadas, assistindo a 8,8\% da população brasileira, e, em fevereiro de 2015, esse percentual de cobertura era de $57 \%^{1,2}$.

Porém, para além da ampliação em números e da melhoria de indicadores de saúde, espera-se que a ESF impulsione, também, um movimento de mudança no modo de se produzir o cuidado em saúde. Nesse sentido, demanda das EqSF envolvidas uma nova organização da dinâmica de trabalho. Cabe à ESF a desafiadora missão de transformar o modelo brasileiro tradicional de assistência à saúde - caracterizado pela centralidade da figura do médico, medicamentoso, curativo, individual e hospitalocêntrico -, em um modelo de assistência coletivo, multi e interdimultiprofissional e baseado na família e no contexto social onde os indivíduos vivem e trabalham. Trata-se de mudar o foco do procedimento para o do sujeito, e de enfrentar o desafio de construir novas práticas sanitárias que levarão a uma assistência à saúde solidária, acolhedora e, consequentemente, mais efetiva e resolutiva ${ }^{3}$.

O setor de saúde brasileiro encontra-se em um cenário de crise no que se refere a sua forma de produzir cuidado, baseando-se em procedimentos orientados por atos prescritivos que valorizam, sobretudo, a dimensão biológica, desconsiderando as subjetividades e os determinantes sociais do processo saúde-doença ${ }^{4}$. O processo de trabalho em saúde, assim como todos os outros, vivenciou profundas transformações com o capitalismo, as quais permitiram o avanço do conhecimento no sentido de preservar a vida, mas, por outro lado, perpetuou a fragmentação do indivíduo e de suas próprias necessidades como objetos de trabalho em saúde ${ }^{5}$.

O trabalho em saúde, devido as suas características específicas de prestação de serviços, detém aspectos bastante específicos, consequentes de sua dimensão intelectual (saberes e autonomia dos seus agentes). As particularidades do objeto de trabalho (as necessidades de saúde) demandadas pelos usuários, e que são interpretadas pelos agentes, atribuem ao trabalho em saúde um caráter reflexivo ${ }^{6}$. A teoria marxista considera três componentes fundamentais do processo de trabalho: a finalidade, o objeto e os instrumentos de trabalho. Esses componentes referem-se a abstrações teóricas pelas quais é possível compreender a realidade das práticas em saúde, onde o trabalho é a base estruturante de sua efetivação?.

Para Marx, o objeto de trabalho é definido como aquilo sobre o qual o trabalho é realizado e que se transforma por meio da ação intencional do trabalhador ${ }^{8}$. Para Mendes-Gonçalves ${ }^{9}$, o objeto de trabalho em saúde não é um objeto natural, porquanto se materializa por meio de um "olhar" que planeja um projeto de transformação. Souza et al. ${ }^{5}$ apontam, a partir de sua revisão teórica, que, no caso do trabalho em saúde, o objeto é a pessoa ou grupos populacionais que, por meio da assistência dos serviços de saúde, transformam-se de acordo com a finalidade do trabalho de manutenção da vida, promoção da saúde e melhoras nas condições de adoecimento e sofrimento. Nesse contexto, é de extrema relevância que se conheça a construção conceitual do objeto de trabalho por trabalhadores da ESF, uma vez que é a partir dela que a matéria a ser transformada será identificada pelos serviços de saúde.

Assim, o objetivo deste estudo foi analisar a percepção de trabalhadores de nível Superior de EqSF consideradas de referência no município de João Pessoa/Paraíba acerca do objeto de trabalho na ESF. 


\section{Metodologia}

Trata-se de um estudo qualitativo, realizado com trabalhadores da saúde de nível Superior de EqSF e do Núcleo de Apoio à Saúde da Família (NASF) do município de João Pessoa/Paraíba. Conforme apontado por Gomes e Silveira ${ }^{10}$, são inegáveis as contribuições dos estudos qualitativos na área da saúde coletiva nas investigações que se propõem a desvelar os modos de sentir, pensar e agir dos sujeitos envolvidos no fenômeno. Além disso, eles sinalizam novas formas de apreender e pensar objetos, sobretudo, quando se considera que visam justificar propostas de intervenções em ações coletivas inscritas no campo da saúde.

A coleta de dados ocorreu no período de agosto a setembro de 2014. Na primeira fase do estudo, foram realizadas entrevistas individuais em uma amostra intencional de quatro Diretores de Distrito Sanitário. A estes sujeitos, foi solicitado que fossem indicadas EqSF que atendessem aos seguintes critérios: (1) possuir a mesma equipe de médico, enfermeiro e cirurgião-dentista pelo tempo mínimo de doze meses; (2) desenvolverem atividades de atenção individual e coletiva; (3) ser considerada EqSF de referência de trabalho na ESF em seu Distrito Sanitário.

A segunda fase consistiu na realização de entrevistas individuais com nove trabalhadores de nível Superior de três Unidades de Saúde da Família - USF (três médicos, três enfermeiros e três cirurgiões-dentistas) e três trabalhadores do NASF, um de cada uma das EqSF, indicados pelos DDS. O tamanho amostral nesta fase, foi adotado o critério de saturação das informações ${ }^{11}$. O roteiro da entrevista apreendeu as percepções e expectativas dos trabalhadores acerca da finalidade, do objeto e instrumentos de trabalho e do trabalho vivo na ESF.

Optou-se por entrevistar trabalhadores de nível Superior de EqSF e NASF consideradas de referência por gestores municipais para rastrear informantes-chave. Com isso, esperou-se a identificação das potencialidades da ESF na reorientação do modelo assistencial. Logo, a construção dos resultados deste artigo a partir da percepção destes trabalhadores pode desvelar a realidade de EqSF que realmente se dedicam aos objetivos deste modelo de atenção.

Foram realizadas doze entrevistas em profundidade, com média de duração de sessenta minutos cada, por um mesmo pesquisador devidamente treinado, no local de trabalho dos participantes, em ambiente reservado, gravadas em áudio, transcritas e submetidas à validação. Foi realizada a leitura das transcrições simultaneamente à audição das gravações para identificação dos possíveis erros da transcrição por um sujeito não envolvido no estudo.

No que se refere às características gerais dos participantes do estudo, 11 eram do sexo feminino (92\%) e com média de idade de 41 anos. Todos os entrevistados desempenhavam suas funções, no momento da entrevista, há pelo menos um ano. Duas EqSF deste estudo obtiveram certificação no Programa Nacional de Melhoria do Acesso e da Qualidade da Atenção Básica (PMAQ-AB), com avaliação muito acima da média, e uma com certificação mediana.

Para a elaboração deste artigo, foi utilizada a dimensão da entrevista que foi disparada pela pergunta: 'Para você, qual o objeto de trabalho na ESF?'. O tratamento dos dados foi realizado por meio da análise do conteúdo, conduzida por um olhar interpretativo do material produzido. Para isso, as transcrições das entrevistas passaram pelos processos de: pré-análise (leitura flutuante do material final e elaboração de indicadores iniciais), exploração do material (definição de categorias e identificação das unidades de registro), tratamento dos resultados, inferência e interpretação (condensação e definição das informações para análise, análise reflexiva e crítica dos resultados) ${ }^{12}$. Da análise de conteúdo emergiram três categorias: o objeto 'usuário'; o objeto 'usuário e sua família' e o objeto 'usuário e sua comunidade'.

Todos os sujeitos convidados a participar do estudo aceitaram, livremente, participar do estudo e assinaram o Termo de Consentimento Livre e Esclarecido. Esta pesquisa foi aprovada pelo Comitê de Ética em Pesquisa do Centro de Pesquisas Aggeu Magalhães/Fiocruz PE, e sua realização foi autorizada pela Secretaria Municipal de Saúde de João Pessoa. 


\section{Resultados e discussão}

\section{O objeto 'usuário'}

Os entrevistados, de maneira geral, apontaram como objeto de trabalho da ESF: o sujeito, o indivíduo e o usuário, que se encontram adscritos em suas USF e que têm necessidades em saúde específicas, o que é previsto pelo Ministério da Saúde ${ }^{13}$. Foi possível constatar que a centralidade no sujeito aponta para uma mudança na forma de se produzir o cuidado, porque os trabalhadores passam a organizar sua abordagem de acordo com o que o usuário necessita, e não mais com o que eles têm a oferecer, abandonando o foco na doença, reflexo da construção histórica da assistência à saúde no Brasil baseada no modelo curativista.

"Quando ele [o usuário] entra aqui na minha sala eu o vejo como um todo. Nessa hora eu posso ver uma manchazinha na pelezinha dele, eu posso ver alguma coisa na unha, no peito. Qualquer coisa eu já registro e já passo para o [nome do médico]. Eu faço anamnese, e eu vejo o que é que ele veio procurar, não é o que eu tenho que oferecer." (T7)

A centralidade nas necessidades do sujeito, na organização, no planejamento e na execução do trabalho entre as ESF participantes deste estudo, é uma forma de mudar o modelo assistencial. Historicamente, as práticas em saúde são construídas a partir da doença instalada abordada de maneira individual e descontextualizada da realidade dos usuários. Ao focar nas demandas singulares, a ESF avança na construção de uma assistência humanizada e capaz de responder às múltiplas necessidades de saúde de sua população adscrita.

Um dos fundamentos e dos princípios da Política Nacional de Atenção Básica (PNAB) envolve a organização e a orientação dos serviços de saúde com base em uma lógica mais centrada nos usuários, o que também é apresentado como uma das características da Atenção Primária à Saúde (APS) por Starfield ${ }^{1,14}$. Assim, a ESF propõe-se a reorganizar o processo de trabalho em saúde, ao alterar o modelo hegemônico de uma assistência tendo como foco a doença, predominantemente biológica, para uma abordagem que responda às necessidades singulares dos usuários, apresentadas nos espaços reais onde os cidadãos constroem sua história e representam seu processo saúde-doença ${ }^{15}$.

A produção do cuidado centrada no usuário pode ser capaz de potencializar a assistência à saúde individual e coletiva, uma vez que aborda aspectos sociais e culturais nos quais os sujeitos estão inseridos, além de suas expectativas, o que os torna coparticipantes do cuidado ${ }^{16,17}$. Assim, o acesso aos serviços da ESF deve ser definido de acordo com as necessidades apresentadas pelos usuários, e não com a oferta de serviços organizada e hierarquizada previamente. Com isso, pode-se reduzir o descompasso entre a oferta assistencial das EqSF e as reais necessidades da população adscrita.

Alguns estudos, a partir da percepção dos usuários, apontam que a inversão do foco da doença para as necessidades dos usuários ainda não é uma realidade entre as EqSF. Franco ${ }^{18}$, ao apreender as percepções dos usuários acerca da ESF em um município de Minas Gerais, verificou que, para os entrevistados, a ESF era um espaço onde é oferecido um atendimento humanizado e acolhedor, porém, ainda centrado na doença. Silva e Fracolli19 ${ }^{19}$ concluem que as práticas, nesse nível de atenção na região da cidade de Alfenas/MG, ainda estão sendo efetivadas de maneira bastante tradicional. São realizadas ações preventivas, centradas na saúde da mulher e da criança, sem espaço para a promoção da saúde e para a diversidade das necessidades da população atendida.

\section{O objeto 'usuário e sua família'}

O reconhecimento do sujeito singular como objeto de trabalho da ESF foi associado à sua abordagem e de sua família. Identificar o núcleo familiar não significou para os entrevistados desconsiderar as necessidades individuais dos usuários, mas assumir a família como componente ativo no processo de produção do cuidado, ampliando tanto o 'olhar', que identifica necessidades, quanto o 'fazer em saúde', que intervém e corresponsabiliza para modificar. 
"Sobre a família, porque a gente é dirigido mais para a família, mas não deixa de ver como um indivíduo, é uma coisa atrelada a outra. Mas o indivíduo como um todo, mas também atrelado à família." (T8)

“É o indivíduo dentro de uma família. Então, a gente tem que pensar ele como um todo. É o indivíduo que está inserido dentro de uma Unidade, que está no seu âmbito familiar, que lida com pessoas membros da sua família, que dependendo da situação por que ele passa, a família pode estar influenciando, enfim a gente faz uma avaliação como um todo." (T12)

Ao incorporarem a relação entre o adoecimento individual e o núcleo familiar, os entrevistados sinalizaram uma ampliação da compreensão do processo saúde-doença. Produzir o cuidado tendo como objeto de intervenção a família é uma forma de reverter o modelo fragmentado, descontextualizado dos aspectos socioculturais e centrado na doença, o que é estimulado desde a implantação da ESF. Nesse contexto, é de mister importância que os conceitos de família, seus arranjos e possibilidades de intervenção, cujo foco seja o núcleo familiar, adotados pelos trabalhadores da ESF, sejam investigados e discutidos em profundidade. Algumas evidências científicas ${ }^{1,14,20-23}$ contribuem para ampliar o debate sobre essas temáticas, conforme apresentado a seguir.

O entendimento de que as situações de adoecimento ou saúde individuais e a família como unidade influenciam-se mutuamente já é consolidado ${ }^{20}$. A PNAB ${ }^{1}$ determina que uma das atribuições comuns a todos os membros das EqSF é o desenvolvimento de práticas cuidadoras familiares por meio de intervenções que influenciem o processo saúde-doença dos usuários e suas famílias. É no contexto familiar onde são vivenciadas e aprendidas questões relacionadas às práticas de cuidado com a saúde e hábitos de vida pela maioria dos usuários.

Uma das características da APS elencada por Starfield é a orientação para a família, compreendendo e analisando o processo saúde-doença no contexto familiar e da rede social ${ }^{14}$. Por meio do estabelecimento de vínculos e do conhecimento da história das famílias dos usuários com necessidades em saúde, os trabalhadores das EqSF podem ampliar sua percepção de demandas, ansiedades, sofrimentos e potenciais que antes seriam ignorados por uma abordagem individualizada ${ }^{20,21}$.

De acordo com a categorização de abordagens da família na ESF proposta por Ribeiro22, foram identificadas, nas falas dos sujeitos entrevistados neste estudo, três concepções de abordagem de família. Na primeira, 'família/indivíduo', a abordagem familiar se concretiza nas necessidades do usuário, foco da atenção. Nesse contexto, o núcleo familiar exerce influência positiva ou negativa no processo saúde-doença de seus membros, desempenha papéis e assume deveres e responsabilidades. Isso requer orientação e treinamento. A segunda é a concepção de 'família/domicílio', em que o ambiente, a infraestrutura material da família, as condições de cuidado desse universo são o foco de atenção. Por fim, a de 'família/indivíduo/domicílio', onde se fundem os sentidos/conteúdo de família/ indivíduo e família/domicílio.

Cabe destacar o apontado por Ribeiro22, que adotar a família como foco da AB não garante que isso se efetive no cotidiano do processo de trabalho na ESF. Os diversos atores envolvidos na produção do cuidado nesse nível de atenção podem ter conceitos de família que ocasionam abordagens distintas. Essa possibilidade de multiplicidade de abordagens impacta no planejamento e na execução do cuidado e a orientação da formação de trabalhadores, e, consequentemente, um entrave na abordagem integral das famílias adscritas, porque essa unidade que será cuidada não é suficientemente identificada.

Silva et al. ${ }^{20}$ realizaram uma revisão integrativa da literatura visando identificar o conceito de família e os fatores associados à abordagem familiar na ESF, e concluíram que não era apresentado o conceito de família e não se encontravam orientações sobre como conduzir a ação profissional diante de questões levantadas sobre a dinâmica familiar nos documentos oficiais que orientam a ESF. Alencar et al. ${ }^{23}$, ao avaliarem o enfoque familiar das ESF em São Luiz/MA, por meio da aplicação do Primary Care Assessment Tool (PCATool) a usuários, gestores e trabalhadores, verificaram que, sob o ponto de vista dos usuários, o interesse por suas condições de vida e de suas famílias ainda não figura entre as questões investigadas pelos profissionais. Já os profissionais e os gestores referiram que essas 
questões são coletadas na maioria das vezes, e que um expressivo percentual de prontuários médicos considerava o núcleo familiar.

A carência de estudos sobre a família na ESF, verificada por Silva et al. ${ }^{20}$, pode ser considerada um sinal de alerta acerca do pouco interesse que ela desperta nos profissionais e na Academia. Associase, à incipiente produção científica sobre o tema, a indefinição conceitual de família e de abordagem familiar nos documentos oficiais; e que existe a possibilidade de que não esteja acontecendo a diferenciação entre o cuidado de um indivíduo doente que tem uma família e o de uma família que tem um indivíduo doente.

Assim, as práticas em saúde podem estar sendo orientadas a partir de conceitos individuais que podem comprometer a efetivação do compromisso de mudança do modelo assistencial proposto pela ESF. Por essa razão, são necessários estudos e debates com as diversas esferas envolvidas. Estes devem visar elaborar um arcabouço teórico para nortear as políticas públicas, as práticas dos trabalhadores das EqSF e os processos de formação e educação permanente.

\section{O objeto 'usuário e sua comunidade'}

A inserção do usuário em sua comunidade também foi apontada como objeto de trabalho pelos entrevistados. O componente 'comunidade' foi associado a uma ampliação da percepção do escopo de questões a serem consideradas pelos trabalhadores no processo saúde-doença dos usuários, que envolvem realidades como a violência e as condições de moradia e de saneamento, conforme segue.

"O foco são as pessoas, é a comunidade. Só que a pessoa como um todo, não um indivíduo isolado, é a pessoa como um todo em sua realidade, como a violência que há no bairro, com a moradia, com o rio enchendo de vez em quando. Não só especificamente como uma pessoa, um indivíduo, é o seu conjunto." (T5)

"Acho que a Saúde da Família, o objeto de trabalho dela é esse caldo social em que essas pessoas estão inseridas, porque se eu trabalhar só o paciente, ele senta e eu dou o remédio, eu não vou resolver o caldo social em que ele está inserido e muito provavelmente não vou resolver o problema que ele me traz. Agora, se eu trabalho no âmbito comunitário a chance de resolver isso é maior." (T9)

Ao associar as necessidades singulares dos usuários ao contexto sociocultural no qual eles estão inseridos, a ESF rompe radicalmente com o modelo hospitalocêntrico de assistência à saúde, e passa a valorizar a multicausualidade. O processo saúde-doença é abordado para além da manifestação de sinais e sintomas determinados por alterações do equilíbrio homestático, e incorpora, na estruturação de suas práticas, o conceito de determinantes sociais que valorizam a relevância de múltiplos aspectos socioculturais. A partir desse olhar, evidencia-se a necessidade de uma abordagem integral da população adscrita à ESF e usuária do SUS e do estabelecimento de parcerias intersetoriais para a adequada assistência à saúde.

Considerando a indissociabilidade entre o indivíduo, a família e a comunidade, presente nas entrevistas deste estudo, novamente é possível identificar a categorização de Ribeiro ${ }^{22}$ para a abordagem familiar na ESF. Aqui estão presentes as duas concepções: 'família/comunidade' e 'família/ risco social'. Na primeira abordagem, consideram-se 'as famílias', definidas por parâmetros gerais de identidade e processo de viver, consequências do contexto, da inserção sociocultural e do ambiente físico da comunidade. As famílias têm responsabilidades com os problemas locais da comunidade, como, por exemplo, a violência e as condições gerais de moradia. Na segunda, a atenção está dirigida às condições de um grupo específico de famílias, geralmente aquelas em exclusão social, que vivem em comunidades periféricas.

A abordagem da concepção do objeto sujeito/comunidade traz à tona o conceito de determinantes sociais da saúde, uma vez que a ESF é colocada como uma ferramenta de orientação do Sistema Único de Saúde que visa atender às necessidades da população a partir do entendimento do direito universal 
à saúde e a relevância do enfrentamento dos determinantes sociais no processo saúde-doença. ${ }^{1}$ Para a Comissão para os Determinantes Sociais da Saúde da Organização Mundial de Saúde, são os determinantes estruturais e as condições de vida das populações que constroem os determinantes sociais da saúde e estão relacionadas ao seu processo de saúde-doença, sendo, assim, responsáveis pela maioria das desigualdades no setor saúde, tanto dentro quanto entre países ${ }^{24}$.

Starfield apresenta que uma das doze características da APS é a orientação para a comunidade ${ }^{14}$. Essa característica se refere: ao contexto local de vida dos usuários, à consciência das necessidades de saúde no âmbito comunitário, o que demanda a necessidade de articulação com outros setores para promover mudanças positivas nas condições de vida e de saúde. De acordo com Arce e Sousa ${ }^{25}$, a ESF, ao considerar a saúde como qualidade de vida e capacidades humanas condicionadas socialmente, contribui fortemente para a construção de um novo modelo assistencial de saúde.

Dowbor e Westphal ${ }^{26}$ investigaram a situação do trabalho com determinantes sociais por EqSF do município de São Paulo/SP, e constataram que as EqSF desenvolviam atividades dirigidas a questões relacionadas a problemas de: educação, saneamento, lazer, alimentação, recursos sustentáveis, distribuição de renda, paz, emprego, habitação, segurança, transporte e ecossistema saudável. Esses autores destacam, também, o potencial da ESF na abordagem dos determinantes sociais da saúde; porém, apontam que é preciso organizar o processo de trabalho de forma que possa romper com as práticas individuais atuais ainda presentes no âmbito da atenção primária à saúde.

Além disso, a abordagem dos determinantes sociais da saúde deve se configurar como uma política de Estado, a qual requer, inclusive, "financiamento, sensibilização e capacitação dos profissionais para que possam desenvolver atividades, avaliar os processos e integrar atividades realizadas por outros setores de governo e pela sociedade civil" 26 (p. 778). O fragmento a seguir ilustra a sensibilidade dos trabalhadores da ESF em considerarem a articulação intersetorial em seu processo de trabalho:

\footnotetext{
“Primeiro tem aquela consulta inicial para a gente identificar o problema e a gente ver a forma como vai trabalhar. Que tem certos procedimentos que dá para fazer no ambiente familiar mas tem procedimentos que a gente precisa fazer no consultório mesmo. Ai a gente vê meio de transporte, como a gente pode estar organizando esse processo." (T7)
}

Nesse contexto, no âmbito da ESF, o trabalho deve ser capaz de: ser articulado, promover ações intersetoriais, fortalecer vínculos com equipamentos sociais, manter, no planejamento, na execução e na avaliação de suas práticas, a associação entre desigualdade social e saúde. Assim, será possível implementar estratégias que fortaleçam a "luta pelos direitos à cidadania, à saúde de qualidade, o acesso à escolarização, a terra, ao trabalho digno, a salário e moradia, entre outros"27 (p. 255), o que foi apontado pelos entrevistados neste estudo.

Silva e Rodrigues ${ }^{28}$ entendem que, para efetivar a intersetorialidade, é fundamental criar estratégias de comunicação que estabeleçam objetivos comuns entre os pontos de atenção à saúde e as áreas relacionadas. Com isso, viabiliza-se o planejamento de atividades que ultrapassem a negociação de ações e a transferência de responsabilidades.

\section{Considerações finais}

A análise do conteúdo das entrevistas com os trabalhadores de nível Superior da ESF do município de João Pessoa permitiu identificar a construção conceitual de seu objeto de trabalho. Evidenciouse o reconhecimento da produção do cuidado centrada no indivíduo a partir de suas necessidades singulares, associado a um núcleo familiar e a uma comunidade. O verificado em João Pessoa destaca o elevado grau de complexidade que orienta o trabalho no âmbito da ESF, onde o objeto de trabalho extrapola a dimensão individual e biologicista, assumindo a determinação social e a sua relação familiar e comunitária.

Embora os resultados apontem para a consolidação de uma mudança de foco assistencial no âmbito da ESF, evidências de estudos anteriores apontam que a centralidade do sujeito contextualizado à sua 
família e a comunidade se apresentou em outras realidades brasileiras com fortes traços de relação entre necessidades em saúde e adoecimento biológico. Essa contradição entre o declarado e a prática cotidiana dos trabalhadores merece ser investigada em profundidade em novos estudos.

Em síntese, os resultados desse estudo contribuíram para a construção de elementos que possibilitam e potencializam o planejamento, organização e execução de práticas de saúde coerentes com os princípios ESF. Além disso, apontaram para a necessidade de reflexões acerca de problemas historicamente consolidados no setor saúde brasileiro, sendo o mais preocupante, conforme verificado, a persistência de características do modelo biomédico na ESF, pelas diversas esferas interessadas na construção e consolidação de um sistema de saúde público, universal, equânime, integral e humanizado.

\section{Colaboradores}

Os autores participaram, igualmente, de todas as etapas de elaboração do artigo.

\section{Referências}

1. Ministério da Saúde (BR). Política Nacional de Atenção Básica. Brasília; 2012.

2. Ministério da Saúde (BR). Sistema de Informação da Atenção Básica: SIAB. Brasília; 2015 [citado 27 Ago 2015]. Disponível em: http://www2.datasus.gov.br/SIAB/index.php.

3. Costa GD, Cotta RMM, Ferreira LSM, Reis JR, Franceschini SCC. Saúde da família: desafios no processo de reorientação do modelo assistencial. Rev Bras Enferm. 2009; 62(1):113-8.

4. Malta DC, Merhy EE. O percurso da linha do cuidado sob a perspectiva das doenças crônicas não transmissíveis. Interface (Botucatu). 2010; 14(34):593-606.

5. Souza SS, Costa R, Shiroma LMB, Maliska JCA, Amadigi FR, Pires EP, et al. Reflexões de profissionais de saúde acerca do seu processo de trabalho. Rev Eletr Enferm. 2010; 12(3):449-55.

6. Peduzzi M. Equipe multiprofissional de saúde: a interface entre trabalho e interação [tese]. Campinas: Universidade Estadual de Campinas, Faculdade de Ciências Médicas;1998.

7. Peduzzi M, Schraiber LB. Processo de trabalho em saúde. In: Pereira IB, Lima JCF, organizadores. Dicionário da educação profissional em saúde [Internet]. 2a ed. Rio de Janeiro: Fundação Oswaldo Cruz, Escola Politécnica Joaquim Venâncio; 2009 [citado 22 Out 2015]. Disponível em: http://www.epsjv.fiocruz.br/dicionario/apresentacao.html.

8. Marx K. O capital. 3a ed. São Paulo: Edipro; 2012.

9. Mendes-Gonçalves RB. Tecnologia e organização social das práticas de saúde. São Paulo: Hucitec; 1994

10. Gomes MHA, Silveira C. Sobre o uso de métodos qualitativos em saúde coletiva, ou a falta que faz uma teoria. Rev Saude Pubica. 2012; 46(1):160-5. 
11. Fontanella BJB, Luchesi BM, Saidel MGB, Ricas J, Turato ER, Melo DG. Amostragem em pesquisas qualitativas: proposta de procedimentos para constatar saturação teórica. Cad Saude Publica. 2011; 27(2):389-94.

12. Bardin L. Análise do conteúdo. São Paulo: Edições 70; 2012.

13. Ministério da Saúde (BR). Portaria GM/MS n 2.027, de 25 de agosto de 2011. Altera a Portaria n 648/GM/MS, de 28 de março de 2006, na parte que dispõe sobre a carga horária dos profissionais médicos que compõem as Equipes de Saúde da Família (ESF) e na parte que dispõe sobre a suspensão do Piso de Atenção Básica (PAB Variável). Diário Oficial da União, Ago 2011. p. 90.

14. Starfield B. Atenção primária: equilíbrio entre necessidades de saúde, serviços e tecnologia. Brasília: UNESCO, Ministério da Saúde; 2004.

15. Assis MMA, Nascimento MAA, Franco TB, Jorge MSA, organizadores. Produção do cuidado no programa saúde da família: olhares analisadores em diferentes cenários. Salvador: EdUfba; 2010.

16. Tavares RP, Costa GC, Falcão MLM, Cristiano PS. A organização do acesso aos serviços de saúde bucal na estratégia de saúde da família de um município da Bahia. Saude Debate. 2013; 37(99):628-37.

17. Silva SA, Baitelo TC, Fracoli LA. Avaliação da atenção primária à saúde: visão de usuários e profissionais sobre a estratégia de saúde da família. Rev Latino-Am Enfermagem. 2015; 23(5):979-87.

18. Franco ECD. A estratégia de saúde da família na perspectiva do usuário. Rev Enferm UFSM. 2012; 2(1):49-58.

19. Silva AS, Fracolli LA. Avaliação da estratégia saúde da família: perspectiva dos usuários em Minas Gerais, Brasil. Saude Debate. 2014; 38(103):692-705.

20. Silva MCLRS, Silva S, Bousso RSA. A abordagem à família na estratégia saúde da família: uma revisão integrativa da literatura. Rev Esc Enferm USP. 2011; 45(5):1250-5.

21. Moimaz SAS, Fadel CB, Yarid SD, Diniz DG. Saúde da família: o desafio de uma atenção coletiva. Cienc Saude Colet. 2011; 16 Supl1:965-72.

22. Ribeiro EM. As várias abordagens da família no cenário do programa/estratégia de saúde da família (PSF). Rev Latino-Am Enfermagem. 2004; 12(4):658-64.

23. Alencar MN, Coimbra LC, Morais PP, Silva AAM, Pinheiro SRA, Queiroz RCS. Avaliação do enfoque familiar e orientação para a comunidade na estratégia saúde da família. Cienc Saude Colet. 2014; 19(2):353-64.

24. Comissão para os Determinantes Sociais da Saúde. Redução das desigualdades no período de uma geração: igualdade na saúde através da acção sobre os seus determinantes sociais: relatório final. Genebra: OMS, 2010 [citado 22 Set 2016]. Disponível em: http://whqlibdoc.who.int/publications/2010/9789248563706_por.pdf.

25. Arce VAR, Sousa MF. Práticas de longitudinalidade no âmbito da estratégia saúde da família no Distrito Federal. Cad Saude Colet. 2014; 22(1):62-8.

26. Dowbor TP, Westphal MF. Determinantes sociais da saúde e o programa saúde da família no município de São Paulo. Rev Saude Publica. 2013; 47(4):781-90.

27. Santana FR, Lima RP, Lopes MM, Fernandes JS, Oliveira NS, Santos WS, et al. Conhecimento de agentes comunitárias de saúde acerca dos determinantes sociais em sua comunidade adscrita. Rev Eletr Enferm. 2012; 14(2):248-56.

28. Silva $K L$, Rodrigues AT. Ações intersetoriais para promoção da saúde na estratégia saúde da família: experiências, desafios e possibilidades. Rev Bras Enferm. 2010; 63(5):762-9. 
Brito GEG, Mendes ACG, Santos Neto PM. El objeto de trabajo en la Estrategia Salud de la Familia. Interface (Botucatu). 2018; 22(64):77-86.

El objetivo de este estudio es analizar la percepción de trabajadores de la Estrategia Salud de la Familia sobre su objeto de trabajo. Se realizaron 12 entrevistas: nueve con trabajadores de tres Equipos Salud de la Familia, considerados como referencia por gestores municipales, y tres con trabajadores del Núcleo de Apoyo a la Salud de la Familia, de ellos, todos con estudios superiores. La transcripción de las entrevistas se sometió al análisis de contenido. Se identificó la construcción conceptual de su objeto de trabajo de los entrevistados. Se trató del reconocimiento de la producción del cuidado centrada en el individuo a partir de sus necesidades singulares, asociado a su familia y comunidad. Ese resultado mostró el elevado grado de complejidad que orienta el trabajo en el ámbito de la Salud de la Familia, en donde el objeto de trabajo extrapola la dimensión individual y biologicista asumiendo la terminación social y su relación familiar y comunitaria.

Palabras clave: Estrategia salud de la familia. Trabajo. Recursos humanos. Análisis cualitativo. 